\title{
On an Algorithm of Billevich for Finding Units in Algebraic Number Fields
}

\author{
By Ray Steiner and Ronald Rudman
}

\begin{abstract}
The well-known algorithm of Billevich for finding units in algebraic number fields is derived by algebraic methods. Some tables of units in cubic and quartic fields are given.
\end{abstract}

I. Introduction. Let $Q(\theta)$ be an algebraic field of degree $n$ over the rationals, and let $Z(\theta)$ be its ring of integers. Suppose the defining polynomial for $Q(\theta)$ has $r$ real roots and $s$ pairs of conjugate complex roots. Then by the famous theorem of Dirichlet and Minkowski, the group of units of $Z(\theta)$ has $r+s-1$ free generators, known as the fundamental units of $Z(\theta)$. However, the proofs of Dirichlet's theorem [6], [10] give no method of finding a set of fundamental units. In fact, if $n>3$ no rapid, efficient method of finding them is known. The best of the known methods of finding units in algebraic number fields of degree $n \geqslant 3$ are those of Voronoi [6], [11] and Billevich [2] , [3], [4]. Voronoì's method is based on the finding of periodic sequences of integers of $Z(\theta)$ called relative minima. Methods of finding the relative minima are given in [6], but these methods have not yet been generalized to fields of degree higher than 3 .

Billevich's method consists of finding the points in a sequence of integers of $Z(\theta)$ called the sequence $\{1\}^{(k)}$. (We call them the $B(1, k)$ numbers.) His method always leads to a system of fundamental units but is very inefficient if the wanted units have large coefficients; e.g., the finding of a unit with seven-digit coefficients may require a full day's computer time for certain fields of the fourth degree. Further, Billevich derives his method in a complicated, geometric manner and gives no algorithm whatever for $r=0$.

The purpose of the present paper is to show how the essential inequalities of Billevich's algorithm can be derived algebraically and to present an algorithm for totally complex algebraic number fields. In addition, we present tables of fundamental units of totally real cubic fields and semireal quartic fields related to Mordell's equation [7], [8], [9].

\section{Billevich's Algorithm,}

A. Theory of the Algorithm. Let us study the problem of finding units from the viewpoint of $n$-dimensional multiplicative lattices [6, Section 1]. We consider an $n$-dimensional multiplicative lattice $\Omega$ in the complex space $K_{n}$ with basis $\omega_{1}, \omega_{2}$, $\ldots, \omega_{n}$, i.e. the collection of all points of the form

Received March 11, 1975; revised September 18, 1975.

AMS (MOS) subject classifications (1970). Primary 12 A45.

Key words and phrases. Algebraic number field, units, Billevich's algorithm, multiplicative lattices, Cramer's rule, fundamental units. 


$$
\omega_{1} x_{1}+\omega_{2} x_{2}+\cdots+\omega_{n} x_{n},
$$

where $x_{1}, x_{2}, \ldots, x_{n}$ range over all possible systems of $n$ rational integers. We assume:

(1) The lattice is irreducible, i.e., no point of $\Omega$ except the origin has a zero coordinate. Then $[6$, Section 4, p. 28$] \Omega$ has the same unit structure as that of a ring of algebraic integers.

(2) The first $r$ coordinates $\omega_{k 1}, \omega_{k 2}, \ldots, \omega_{k r}$ of each basis point $\omega_{k}$ are real, while the remaining $n-r=2 t$ coordinates are pairwise conjugate complex numbers:

$$
\begin{aligned}
\omega_{k, r+1} & =\rho_{k 1}+i \sigma_{k 1}, \\
\omega_{k, r+2} & =\rho_{k 1}-i \sigma_{k 1}, \\
& \vdots \\
\omega_{k, n-1} & =\rho_{k t}+i \sigma_{k t}, \\
\omega_{k, n} & =\rho_{k t}-i \sigma_{k t} .
\end{aligned}
$$

In the $n$-dimensional signature space $R_{n, t}$ corresponding to $K_{n}[6$, Section 1], we can find a lattice $[\omega]$ which is also multiplicative, has all its coordinates real, and corresponds to $\Omega$. To each basis point $\omega_{k}$, there corresponds the point $\omega_{k 1}, \omega_{k 2}, \ldots$, $\omega_{k r}, \rho_{k 1}, \sigma_{k 1}, \ldots, \rho_{k t}, \sigma_{k t}$. Every point $M$ of $[\omega]$ has coordinates equal to the values of the forms

$$
\begin{aligned}
& \xi_{i}=\omega_{1 i} x_{1}+\omega_{2 i} x_{2}+\cdots+\omega_{n i} x_{n} \quad(i=1,2, \ldots, r), \\
& \eta_{i}=\rho_{1 i} x_{1}+\rho_{2 i} x_{2}+\cdots+\rho_{n i} x_{n} \text {, } \\
& \zeta_{i}=\sigma_{1 i} x_{1}+\sigma_{2 i} x_{2}+\cdots+\sigma_{n i} x_{n} \quad(i=1,2, \ldots, t) .
\end{aligned}
$$

Now let us recall some definitions [6, Sections 1 and 4].

Definition 1. The parameters of a point $M$ of $[\omega]$ are

$$
\left|\xi_{1}\right|,\left|\xi_{2}\right|, \ldots,\left|\xi_{r}\right|, \quad \eta_{1}^{2}+\zeta_{1}^{2}, \ldots, \eta_{t}^{2}+\zeta_{t}^{2} .
$$

Definition 2. The normed body of $M$ is the collection of all points of $R_{n, t}$ whose parameters do not exceed the parameters of $M$.

The normed body of $M$ is clearly a convex body with center at the origin. If, for example, $n=3, r=3, t=0$, it is a rectangular parallepiped with one of its vertices at $M$ and its faces parallel to the coordinate planes. If $n=3, r=1, t=1$, it is a right circular cylinder with center at the origin and with $M$ lying on the circumference of one of the bases [6, p. 28].

Definition 3. The norm of $M$ is defined by

$$
N(M)=\xi_{1} \xi_{2} \xi_{3} \cdots \xi_{r}\left(\eta_{1}^{2}+\zeta_{1}^{2}\right) \cdots\left(\eta_{t}^{2}+\zeta_{t}^{2}\right) .
$$

It is well known [6, Section 1] that $N(M)$ is a rational integer.

Definition 4. $M$ is a relative minimum of a lattice if it is nonzero and if there are no points of the lattice except the origin within its normed body.

We shall assume from now on that $[\omega]$ contains the point 1 . Then $[6, p .28]$, 1 is a relative minimum of the lattice, provided $[\omega]$ is maximal. 
Definition 5. The region (1) $)^{(k)}$ is the region of $R_{n, t}$ in which the parameters of each point with the exception of the $k$ th parameter are not greater than unity, while the $k$ th can have any value.

Thus, if $n=3, r=3, t=0,(1)^{(k)}$ is an infinite four sided prism with edges parallel to $\xi_{i}$, while if $n=3, r=1, t=1, k=1$, it is an infinite circular cylinder with generator parallel to the $\xi_{1}$ axis $[2$, p. 124].

The point 1 of $[\omega]$ lies on the edge of $(1)^{(k)}$. We shall begin to increase the $k$ th parameter of the point 1 , while leaving all the remaining parameters unchanged. Then the volume of the normed body will increase, and when it exceeds $2^{n}$ times the volume of the basic parallepiped of the lattice, we find on applying Minkowski's lattice point theorem, at least two points of the lattice, symmetric with respect to the origin, and having their corresponding parameters equal. As we further increase the size of the normed body, we find more and more points of $[\omega]$ in it. Since $[\omega]$ is irreducible, there will be no points in it with equal $k$ th coordinates. However $[6$, p. 31], there may be points with the same $k$ th parameter. From the set of points with equal $k$ th parameters, we choose the point with smallest absolute norm (or any of the points with smallest absolute norm if several exist) and arrange the points obtained according to the size of their $k$ th parameters. We obtain a sequence of points called the $B(1, k)$ numbers. The points themselves will be called $\lambda_{1}, \lambda_{2}, \ldots$. We also include 1 in the sequence. Finally we note that all $B(1, k)$ numbers belong to the region $(1)^{(k)}$.

B. Calculation of the $B(1,1)$ Numbers for Totally Real Lattices. Suppose $r=n$, $t=0, k=1$. Then the forms (1) are

$$
\xi_{i}=\omega_{1 i} x_{1}+\omega_{2 i} x_{2}+\cdots+\omega_{n i} x_{n} \quad(i=1,2, \ldots, n) .
$$

The coefficients of all these forms are real numbers. Also, we agree to only include points with positive first coordinate in $B(1,1)$. If by our technique below, we find a point with negative $\xi_{1}$ we replace that point by its negative and include the latter in $B(1,1)$.

The conditions that a point $M$ be a $B(1,1)$ number are

$$
\begin{array}{r}
\omega_{11} x_{1}+\cdots+\omega_{n 1} x_{1}>1 \\
-1<\omega_{12} x_{1}+\cdots+\omega_{n 2} x_{2}<1
\end{array}
$$

$$
-1<\omega_{1 n} x_{1}+\cdots+\omega_{n n} x_{n}<1
$$

Let us examine only the last $n-1$ of these conditions. If we find $x_{1}, \ldots, x_{n}$, not all zero, satisfying these, the first will automatically hold, since the product of all the forms is a rational integer.

Let us now study the system

$$
\begin{gathered}
\omega_{12} x_{1}+\cdots+\omega_{n 2} x_{n}<1, \\
\vdots \\
\omega_{1 n} x_{n}+\cdots+\omega_{n n} x_{n}<1 .
\end{gathered}
$$


We assume a value for $x_{1}$, say $p_{1}$. Then

$$
\begin{gathered}
\omega_{22} x_{2}+\cdots+\omega_{n 2} x_{n}<1-\omega_{12} p_{1}, \\
\omega_{23} x_{2}+\cdots+\omega_{n 3} x_{n}<1-\omega_{13} p_{1}, \\
\vdots \\
\omega_{2 n} x_{2}+\cdots+\omega_{n n} x_{n}<1-\omega_{1 n} p_{1} .
\end{gathered}
$$

Since all these inequalities are strict we may convert them to equations by adding positive slack variables $s_{1}, s_{2}, \ldots, s_{n-1}$ to each inequality. Since each of the last $n-1$ forms of (2) is between -1 and 1 , each $s_{i}$ is between 0 and 2. Thus,

$$
\begin{gathered}
\omega_{22} x_{2}+\cdots+\omega_{n 2} x_{n}=1-\omega_{12} p_{1}-s_{1}, \\
\omega_{23} x_{2}+\cdots+\omega_{n 3} x_{n}=1-\omega_{13} p_{1}-s_{2}, \\
\vdots \\
\omega_{2 n} x_{2}+\cdots+\omega_{n n} x_{n}=1-\omega_{1 n} p_{1}-s_{n-1} .
\end{gathered}
$$

Since $\left(\omega_{1}, \ldots, \omega_{n}\right)$ form a basis for $[\omega]$, any subset of them is linearly independent; and thus, the determinant of (3) is nonzero. So we can solve (3) for $x_{2}$ by Cramer's rule:

$$
x_{2}=\left|\begin{array}{ccc}
1-\omega_{12} p_{1}-s_{1} & \cdots & \omega_{n 2} \\
1-\omega_{13} p_{1}-s_{2} \cdots & \omega_{n 3} \\
\vdots & \\
1-\omega_{1 n} p_{1}-s_{n-1} & \cdots & \omega_{n n}
\end{array}\right| /|| \begin{array}{cc}
\omega_{22} \cdots \omega_{n 2} \\
\vdots \\
\omega_{2 n} \cdots \omega_{n n}
\end{array} \mid
$$

On setting $x_{2}=p_{2}$ we get

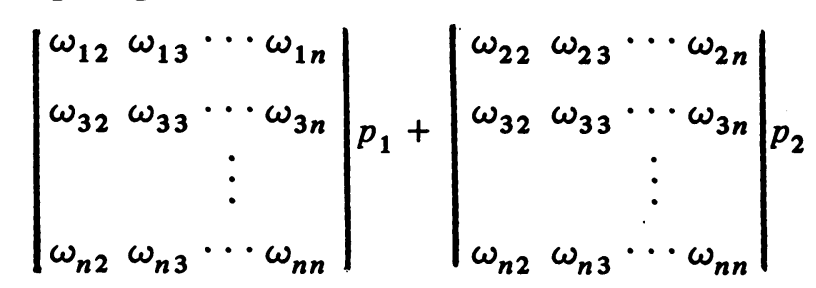

$$
\begin{aligned}
& =\left|\begin{array}{cccc}
1 & \omega_{32} & \cdots & \omega_{n 2} \\
1 & \omega_{33} & \cdots & \omega_{n 3} \\
\vdots & \\
1 & \omega_{3 n} & \cdots & \omega_{n n}
\end{array}\right|-\left|\begin{array}{cccc}
s_{1} & \omega_{32} & \cdots & \omega_{n 2} \\
s_{2} & \omega_{33} & \cdots & \omega_{n 3} \\
& \vdots \\
s_{n-1} & \omega_{3 n} & \cdots & \omega_{n n}
\end{array}\right| \\
& =\left(1-s_{1}\right) A_{11}+\left(1-s_{2}\right) A_{12}+\cdots+\left(1-s_{n-1}\right) A_{1, n-1} \text {, }
\end{aligned}
$$

where $A_{11}, \ldots, A_{1, n-1}$ are the minors of the first row of either determinant of the left-hand side of (4).

Finally, applying the triangle inequality, and using the fact that $s_{1}, \ldots, s_{n-1}$ 
are all positive and less than 2 , we get

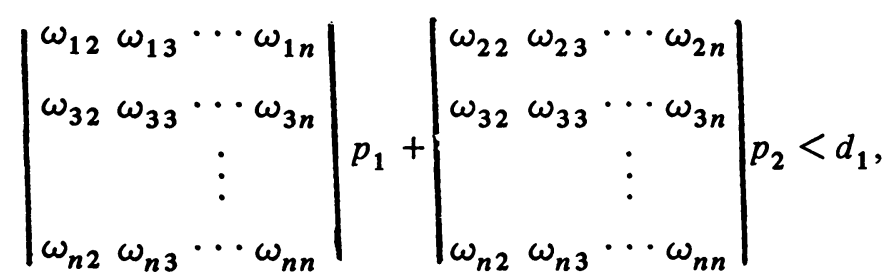

where $d_{1}=\sum_{i=1}^{n}\left|A_{1 i}\right|$. Similarly, using the other side of the inequalities, we find

$$
-d_{1}<\left|\begin{array}{c}
\omega_{12} \cdots \omega_{1 n} \\
\vdots \\
\omega_{n 2} \cdots \omega_{n n}
\end{array}\right| p_{1}+\left|\begin{array}{cc}
\omega_{22} \cdots & \omega_{2 n} \\
\vdots \\
\omega_{n 2} \cdots \\
\cdots
\end{array}\right| p_{2}<d_{1}
$$

which proves Billevich's inequality [2, p. 126].

Thus, the second step in the algorithm is to calculate all possible values of $p_{2}$ satisfying (5). Next, we pick $p_{1}$ and a possible value of $p_{2}$. Then the value of $p_{3}$ may be found from

$$
-d_{2}<\left|\begin{array}{c}
\omega_{13} \cdots \\
\vdots \\
\omega_{n 3} \cdots \\
\cdots
\end{array}\right| p_{1 n}+\left|\begin{array}{ccc}
\omega_{23} & \cdots & \omega_{2 n} \\
& \vdots \\
\omega_{n 3} & \cdots & \omega_{n n}
\end{array}\right| p_{2}+\left|\begin{array}{c}
\omega_{33} \cdots \omega_{3 n} \\
\vdots \\
\omega_{n 3} \cdots \omega_{n n}
\end{array}\right| p_{3}<d_{2},
$$

where $d_{2}=\Sigma_{i=1}^{n-2}\left|A_{2 i}\right|$ and $A_{21}, \ldots, A_{2, n-2}$ are the minors of the first row of any of the determinants in (6). Finally, having found a system of values $p_{1}, \ldots, p_{n-1}$, we find $p_{n}$ from

$$
-1<\omega_{1 n} p_{1}+\cdots+\omega_{n n} p_{n}<1 .
$$

Next, suppose we wish to determine all points in the sequence $B(1,1)$ with $\xi_{1}<$ h. By applying the above technique to the system

$$
\begin{gathered}
\omega_{11} p_{1}+\omega_{21} p_{2}+\cdots+\omega_{n 1} p_{n}<h, \\
\vdots \\
\left|\omega_{1 n} p_{1}+\omega_{2 n} p_{2}+\cdots+\omega_{n n} p_{n}\right|<1,
\end{gathered}
$$

we find

$$
\left|p_{1}\right|<\frac{1}{|\Delta|}\left|A_{1}\right| h+\left|A_{2}\right|+\cdots+\left|A_{n}\right|,
$$

where $\Delta$ is the determinant of the system (1) and $A_{1}, A_{2}, \ldots, A_{n}$ are the minors of its first column. This result will be of the utmost importance in future papers when we modify Billevich's method to find relative minima.

C. Calculation of the Sequence $B(1, k)$ for Partially Real and Totally Complex Latices. The extension of the method of the previous section to partially real and totally complex lattices presents no essential difficulty. First of all, if the lattice is partially real and $k \leqslant r$, we proceed as follows: 
We have the conditions

$$
\begin{gathered}
\left|\xi_{1}\right|<1, \ldots,\left|\xi_{k-1}\right|<1, \quad\left|\xi_{k}\right|>1, \ldots,\left|\xi_{r}\right|<1, \\
\left|\eta_{1}^{2}+\zeta_{1}^{2}\right|<1, \ldots,\left|\eta_{t}^{2}+\zeta_{t}^{2}\right|<1 .
\end{gathered}
$$

We ignore $\left|\xi_{k}\right|>1$, and we replace the other constraints by

$$
\begin{gathered}
\left|\xi_{1}\right|<1, \ldots,\left|\xi_{k-1}\right|<1, \quad\left|\xi_{k+1}\right|<1, \ldots,\left|\xi_{r}\right|<1, \\
\left|\eta_{1}\right|<1,\left|\zeta_{1}\right|<1, \ldots,\left|\eta_{t}\right|<1,\left|\xi_{t}\right|<1 .
\end{gathered}
$$

In virtue of the result on p. 98 of [5] we may then use the method of subsection $B$ to find a point satisfying (10) and for each such point computed we check whether the quadratic constraints of (9) hold. If they do, we include this point in the sequence $B(1, k)$. Thus, in this case the method of procedure is essentially the same as that of part B. The most difficult cases occur when [ $\omega]$ is partially real and $k>r$ or when the lattice is totally complex. In this case we still replace all quadratic forms by linear forms and ignore all constraints with a greatẹr than sign. But in all the above cases we had $n-1$ inequalities and $n$ variables. Here we may have $n+2$ variables, so we must choose two of them and proceed as above. Let us illustrate this by stating the algorithm explicitly for some fields of small degree.

(1) The case $n=3, r=1, t=1, k=2$. This is the simplest case. We have

$$
\begin{aligned}
& \xi_{1}=\omega_{11} x_{1}+\omega_{12} x_{2}+x_{3}, \quad\left|\xi_{1}\right|<1, \\
& \eta_{1}=\rho_{1} x_{1}+\rho_{2} x_{2}+x_{3}, \\
& \xi_{1}=P_{1} x_{1}+P_{2} x_{2}, \quad \eta_{1}^{2}+\xi_{1}^{2}>1 .
\end{aligned}
$$

Here we choose values of $x_{1}$ and $x_{2}$, i.e., $p_{1}$ and $p_{2}$ then find $p_{3}$ from

$$
-1-\omega_{11} p_{1}-\omega_{12} p_{2} \leqslant p_{3} \leqslant 1-\omega_{11} p_{1}-\omega_{12} p_{2} \text {. }
$$

(2) The case $n=4, r=0, t=2, k=1$. Now we have

$$
\eta_{1}^{2}+\zeta_{1}^{2}>1, \quad\left|\eta_{2}\right|<1, \quad\left|\zeta_{2}\right|<1,
$$

i.e., since $\omega_{4}=(1,0,1,0)$,

$$
\begin{aligned}
& -1 \leqslant \rho_{12} x_{1}+\rho_{22} x_{2}+\rho_{32} x_{3}+x_{4} \leqslant 1, \\
& -1 \leqslant P_{12} x_{1}+P_{22} x_{2}+P_{32} x_{3} \leqslant 1 .
\end{aligned}
$$

Here we choose $p_{1}$ and $p_{2}$, and we can find $p_{3}$ from the second inequality, $p_{4}$ from the first.

In a similar fashion we may state the algorithm for all the other cases.

D. Calculation of the Fundamental Units of $[\omega]$. From now on, the units of $[\omega]$ in the sequence $B(1, k)$ will be called $B(1, k)$ units. The following theorems are well known:

THEOREM $1[6$, p. 30]. If $r+t \geqslant 1$, there exist infinitely many $B(1, k)$ units.

THEOREM 2 [2, p. 127]. If $r+t=2$, there is one fundamental unit and the first $B(1, k)$ unit after the point 1 may be taken as the fundamental unit of $[\omega]$. 
If $r+t=3$, there are two fundamental units of $[\omega]$ and they may be found in either of two ways:

THEOREM $3[2, \mathrm{p} .128]$. The first $B(1, k)$ unit, $\epsilon_{1}$, after 1 may be taken as the first fundamental unit of $[\omega]$. For the second fundamental unit one may take the next $B(1, k)$ unit, $\epsilon_{2}$, which has one of its parameters, with the exception of the kth, greater than the corresponding parameter of $\epsilon_{1}$.

THEOREM 4. Let $\epsilon_{1}, \epsilon_{2}, \epsilon_{3}$ be the first $B(1,1), B(1,2)$ and $B(1,3)$ units after the point 1. Any pair of these units forms a fundamental system of units of $[\omega]$.

Theorem 4 was also proved by Berwick [1].

If $r+t>3$, the rules for finding the fundamental units become quite complicated. As an illustration, we state, the following theorem for $r+t=4$, [2], which was later generalized by Billevich to arbitrary lattices [4] .

THEOREM 5. If $r+t=4$ there are three fundamental units, $\epsilon_{1}, \epsilon_{2}, \epsilon_{3}$. They can be calculated as follows: $\epsilon_{1}$ may be taken as the first $B(1, k)$ unit after the point 1. $\epsilon_{2}$ may be taken as the next $B(1, k)$ unit such that at least one of its parameters, with the exception of the kth, is larger than the corresponding parameter of $\epsilon_{1}$.

Let $\mu$ be the first $B(1, k)$ unit after $\epsilon_{1}, \epsilon_{2}$ such that none of the determinants

$$
\Delta=\left|\begin{array}{lll}
\log \left|\mu_{l}\right| & \log \left|\mu_{m}\right| & \log \left|\mu_{p}\right| \\
\log \left|\epsilon_{1 l}\right| & \log \left|\epsilon_{1 m}\right| & \log \left|\epsilon_{1 p}\right| \\
\log \left|\epsilon_{2 l}\right| & \log \left|\epsilon_{2 m}\right| & \log \left|\epsilon_{2 p}\right|
\end{array}\right|
$$

is 0 , where $k, l, m, p=1,2,3,4, k \neq l \neq m \neq p$, and the subscripts denote conjugates. If $\mu_{k}>\epsilon_{1 k} \epsilon_{2 k}$ and in the sequence $B(1, k)$ there are either no units at all between 1 and the first point whose kth parameter exceeds $\sqrt{\epsilon_{1 k} \epsilon_{2 k} \mu_{k}}$ or there are no units $\mu_{i}$ such that

$$
0 \neq \Delta_{i}=\left|\begin{array}{lll}
\log \left|\mu_{l}\right| & \log \left|\mu_{m}\right| & \log \left|\mu_{p}\right| \\
\log \left|\epsilon_{1 l}\right| & \log \left|\epsilon_{1 m}\right| & \log \left|\epsilon_{1 p}\right| \\
\log \left|\epsilon_{2 l}\right| & \log \left|\epsilon_{2 m}\right| & \log \left|\epsilon_{2 p}\right|
\end{array}\right|
$$

has the same sign as $\Delta$ and $\left|\Delta_{i}\right|<|\Delta|$, then $\epsilon_{3}$ may be chosen as $\mu$. If there are such units $\mu_{i}$, then for each $\mu_{i}$ we must compute a number $d_{i}$ equal to $\min \left(\left|\Delta_{i}\right|,\left|\Delta-\Delta_{i}\right|\right)$. Let $d^{*}$ be the smallest such $d_{i}$ and $\mu^{*}$ the corresponding $\mu_{i}$. If $\mu^{*}$ corresponds to some $\left|\Delta_{i}\right|$, then we may choose $\epsilon_{3}=\mu^{*}$. If it corresponds to some $\left|\Delta-\Delta_{i}\right|$, then $\epsilon_{3}=$ $\epsilon_{1} \epsilon_{2} \mu\left(\mu^{*}\right)^{-1}$.

As is obvious, the statement of Theorem 5 is quite complicated. A simpler theorem would obviously be desirable. Also, the question of the fundamental character of the first $B(1, k)$ units for different $k$ remains open, though it can be proved that these units are always independent.

III. Numerical Examples. 1. Find the fundamental units of the field $Q(\theta)$, where $\theta^{3}=6 \theta+2$. 
Solution. By any standard method of finding an integral basis, we find such a basis to be $\left(\theta^{2}, \theta, 1\right)$, and that

$$
\begin{aligned}
& \xi_{1}=5.115749 x_{1}-2.226180 x_{2}+x_{3}, \\
& \xi_{2}=0.115516 x_{1}-0.339877 x_{2}+x_{3}, \\
& \xi_{3}=6.768734 x_{1}+2.601679 x_{2}+x_{3} .
\end{aligned}
$$

(Here and elsewhere in this section all irrational numbers have been rounded to 6 decimal places.)

Let us now find the $B(1,1)$ numbers. We use a slightly modified form of the algorithm presented above. We have $\left|\xi_{1}\right|>1,\left|\xi_{2}\right|<1,\left|\xi_{3}\right|<1$, and

$$
\begin{aligned}
& -1<\omega_{12} x_{1}+\omega_{22} x_{2}+x_{3}<1, \\
& -1<\omega_{13} x_{1}+\omega_{23} x_{2}+x_{3}<1,
\end{aligned}
$$

which yields

$$
-2<\left(\omega_{12}-\omega_{13}\right) x_{1}+\left(\omega_{22}-\omega_{23}\right) x_{2}<2
$$

If we now fix the value of $x_{1}$, we get a bound for $x_{2}$ in terms of $x_{1}$. We find that

$$
\omega_{12}-\omega_{13} \doteq-0.665322, \quad \omega_{22}-\omega_{23} \doteq-0.294156 \text {. }
$$

Further, given the values of $x_{1}$ and $x_{2}$, we can find $x_{3}$ from (11). If we let $x_{1}=0$, the only $B(1,1)$ number we find is 1 . If we let $x_{1}=1$, the first $B(1,1)$ number we find is $-1-2 \theta+\theta^{2}$, which has norm 1 and $\xi_{1} \doteq 8.639354$. Thus, $\epsilon_{1}=-1-2 \theta+\theta^{2}$.

To find the $B(1,2)$ numbers, we now set $\left|\xi_{2}\right|>1,\left|\xi_{1}\right|<1,\left|\xi_{3}\right|<1$ and repeat the above process. We obtain the following results:

\begin{tabular}{lr}
\multicolumn{1}{c}{ Point } & Norm \\
1 & 1 \\
$-6+\theta^{2}$ & 4 \\
$-18-\theta+3 \theta^{2}$ & -2 \\
$-17-\theta+3 \theta^{2}$ & -7 \\
$-36-2 \theta+6 \theta^{2}$ & -16 \\
$-35-2 \theta+6 \theta^{2}$ & -3 \\
$-47-3 \theta+8 \theta^{2}$ & 21 \\
$-53-3 \theta+9 \theta^{2}$ & 1
\end{tabular}

Thus, $\epsilon_{2}=53+3 \theta-9 \theta^{2}$.

2. Find the fundamental units of the field $Q(\theta)$, where $\theta^{4}=6 \theta^{2}+6 \theta+3$.

Solution. An integral basis of the field is $\left(\theta^{3}, \theta^{2}, \theta, 1\right)$, and we find the roots of the defining equation to be

$$
\begin{aligned}
\theta & \doteq-1.920785, \quad \theta^{\prime} \doteq 2.902320, \\
\theta^{\prime \prime} & =-0.490768+0.545243 i, \quad \vec{\theta}^{\prime \prime}=-0.490768-0.545243 i .
\end{aligned}
$$

Thus,

$$
\xi_{1}=-7.086570 x_{1}+3.689414 x_{2}-1.920785 x_{3}+x_{4},
$$




$$
\begin{aligned}
& \xi_{2}=24.447575 x_{1}+8.423460 x_{2}+2.902320 x_{3}+x_{4}, \\
& \eta_{1}=0.319500 x_{1}-0.056437 x_{2}-0.490768 x_{3}+x_{4}, \\
& \zeta_{1}=0.231875 x_{1}-0.535175 x_{2}+0.545243 x_{3} .
\end{aligned}
$$

To find the $B(1,1)$ numbers we set $\left|\xi_{1}\right|>1,\left|\xi_{2}\right|<1,\left|\eta_{1}\right|<1,\left|\zeta_{1}\right|<1$ and find $d_{1} \doteq 4.483573, \Delta_{1} \doteq-12.368891, \Delta_{2}=-6.43949884$, where $\Delta_{i}$ is the coefficient of $p_{i}$ in (*). The first $B(1,1)$ number we find is $-2-2 \theta-2 \theta^{2}+\theta^{3}$, which has norm 1 and $\xi_{1}<0$. Thus, $\epsilon_{1}=2+2 \theta+2 \theta^{2}-\theta^{3}$.

To find the $B(1,2)$ numbers, we now set $\left|\xi_{2}\right|>1,\left|\xi_{1}\right|<1,\left|\eta_{1}\right|<1,\left|\zeta_{1}\right|<1$, and repeat the algorithm. We find that $\epsilon_{2}=2+5 \theta+6 \theta^{2}+2 \theta^{3}$.

IV. Computational Results. As we indicated in the introduction, Billevich's algorithm is very efficient if the fundamental units of the field have small coefficients. However, there are fields [11] for which we could never find the fundamental units, even after using many centuries of computer time. But we recommend the algorithm as the first step in searching for the fundamental units of a given field, as it is quite powerful.

We have, at present, programmed the method only for cubic fields and semireal quartic fields. The programming for these fields is quite straightforward, it being merely necessary to find the roots of the defining equation, set up the inequalities (2), set a range for $p_{1}$ and then calculate the determinants of $(*)$ to find bounds on the remaining variables. However, it is impractical to use (*) to search for $B(1, k)$ numbers in fields of large degree, since this involves the evaluation of many large determinants. This not only slows the computer program exponentially, but also leads to a great loss of significance. In a future paper, we shall show that Billevich's basic idea, that of bounding one variable and using this bound to find bounds of the remaining variables, may be developed from a different viewpoint which involves practically no evaluation of determinants.

In conclusion, we present the following tables of units of cubic and semireal quartic fields associated with the Mordell Diophantine equation $y^{2}+k=x^{3}$. Table I is a list of the fundamental units of the fields in a table of Hemer [8], while Table II is a list of fundamental units for some of the quartic rings $Z\left[1, \theta, \theta^{2}, \theta^{3}\right]$ of Table 10 of [9].

TABLE I. Some units of totally real cubic fields $K=Q(\theta) ; \theta^{3}=q \theta+n$.

$\begin{array}{cccc}q & n & \begin{array}{c}\text { Integral basis } \\ \text { of } Q(\theta)\end{array} & \begin{array}{c}\text { Fundamental units of } \\ Q(\theta)\end{array} \\ 6 & 2 & 1, \theta, \theta^{2} & -1-2 \theta+\theta^{2} ; \\ 12 & 14 & 1, \theta, \theta^{2} & 53+3 \theta-9 \theta^{2} \\ & & & -11-5 \theta+2 \theta^{2} \\ 9 & 6 & 1, \theta, \theta^{2} & 81+11 \theta-8 \theta^{2} \\ & & & -35-39 \theta+15 \theta^{2} \\ & & & 289+24 \theta-34 \theta^{2}\end{array}$


TABLE I (continued)

$q$

9

15

9

12

12

63

18

27

12

12

15

12

24

18

60

21
Integral basis of $Q(\theta)$

$1, \theta,\left(\theta+\theta^{2}\right) / 2$

$1, \theta, \theta^{2}$

$1, \theta, \theta^{2}$

$1, \theta, \theta^{2} / 2$

$1, \theta, \theta^{2}$

$1, \theta, \theta^{2}$

$1, \theta, \theta^{2}$

$1, \theta, \theta^{2}$

$1, \theta, \theta^{2}$

$1, \theta, \theta^{2} / 2$

$1, \theta, \theta^{2}$

16

2

$1, \theta, \theta^{2}$

$1, \theta, \theta^{2}$

178
24

32

42
Fundamental units of

$Q(\theta)$

$\left(-2-3 \theta+2 \theta^{2}\right) / 2$

$\left(62+3 \theta-14 \theta^{2}\right) / 2$

$-221-87 \theta+31 \theta^{2}$

$161+21 \theta-13 \theta^{2}$

$-59-245 \theta+85 \theta^{2}$

$161+4 \theta-18 \theta^{2}$

$\left(-14-8 \theta+3 \theta^{2}\right) / 2$

$11+\theta-\theta^{2}$

$-3-3 \theta+\theta^{2}$

$11+\theta-\theta^{2}$

$-685-81 \theta+17 \theta^{2}$

$13319+1341 \theta-305 \theta^{2}$

$-9-3 \theta+\theta^{2}$

$225+26 \theta-15 \theta^{2}$

$-9739-2242 \theta+648 \theta^{2}$

$83+10 \theta-4 \theta^{2}$

$-53-89 \theta+28 \theta^{2}$

$47+2 \theta-4 \theta^{2}$

$\left(-6-16 \theta+5 \theta^{2}\right) / 2$

$107+3 \theta-9 \theta^{2}$

$-5-3 \theta+\theta^{2}$

$1880891+162230 \theta$

$-138098 \theta^{2}$

$-3-17 \theta+5 \theta^{2}$

$431+6 \theta-36 \theta^{2}$

$-2353-658 \theta+192 \theta^{2}$

$115+13 \theta-6 \theta^{2}$

$-89-39 \theta+12 \theta^{2}$

$31+3 \theta-2 \theta^{2}$

$-22181-2784 \theta+589 \theta^{2}$

$211+21 \theta-5 \theta^{2}$

$-47-17 \theta+5 \theta^{2}$

$\left(266+27 \theta-30 \theta^{2}\right) / 2$ 
TABLE I (continued)

\begin{tabular}{|c|c|c|c|}
\hline$q$ & $n$ & $\begin{array}{l}\text { Integral basis } \\
\quad \text { of } Q(\theta)\end{array}$ & $\begin{array}{l}\text { Fundamental units of } \\
\qquad Q(\theta)\end{array}$ \\
\hline 15 & 12 & $1, \theta, \theta^{2}$ & $\begin{array}{l}-553-528 \theta+156 \theta^{2} \\
901+53 \theta-63 \theta^{2}\end{array}$ \\
\hline 18 & 22 & $1, \theta, \theta^{2}$ & $\begin{array}{l}-1679-879 \theta+259 \theta^{2} \\
16417+1386 \theta-1017 \theta^{2}\end{array}$ \\
\hline 15 & 10 & $1, \theta, \theta^{2}$ & $\begin{array}{l}-9579-11618 \theta+3336 \theta^{2} \\
189+9 \theta-13 \theta^{2}\end{array}$ \\
\hline 30 & 60 & $1, \theta, \theta^{2} / 2$ & $\begin{array}{l}-241-56 \theta+15 \theta^{2} \\
211+23 \theta-9 \theta^{2}\end{array}$ \\
\hline
\end{tabular}

TABLE II. A list of fundamental units of some semireal quartic rings $Z\left(1, \theta, \theta^{2}, \theta^{3}\right) \cdot \theta^{4}=q \theta^{2}+n \theta+r$.

\begin{tabular}{|c|c|c|c|}
\hline$q$ & $n$ & $r$ & Fundamental units \\
\hline 30 & 80 & 75 & $\begin{array}{l}16368481+19932975 \theta+9559246 \theta^{2} \\
+1444393 \theta^{3} \\
5179+4138 \theta+964 \theta^{2}-258 \theta^{3}\end{array}$ \\
\hline 102 & 544 & 867 & $\begin{array}{l}708301+323897 \theta+28214 \theta^{2}-4801 \theta^{3} \\
69820849+49475087 \theta+12229096 \theta^{2} \\
+992385 \theta^{3}\end{array}$ \\
\hline 6 & 6 & 3 & $\begin{array}{l}2+5 \theta+6 \theta^{2}+2 \theta^{3} \\
2+2 \theta+2 \theta^{2}-\theta^{3}\end{array}$ \\
\hline 6 & 2 & 3 & $\begin{array}{l}149+155 \theta+356 \theta^{2}+133 \theta^{3} \\
14+4 \theta+26 \theta^{2}-11 \theta^{3}\end{array}$ \\
\hline 60 & 240 & 300 & $\begin{array}{l}223+202 \theta+66 \theta^{2}+7 \theta^{3} \\
15013+8880 \theta+1151 \theta^{2}-240 \theta^{3}\end{array}$ \\
\hline
\end{tabular}

Mathematics Department Bowling Green University Bowling Green, Ohio 43403

13151 Elgin

Huntingdon Woods, Michigan 48070

1. W. E. H. BERWICK, "Algebraic number fields with two independent units," Proc. London Math. Soc., v. 34 (2), 1932, pp. 360-378.

2. K. K. BILLEVIČ, "On units of algebraic fields of third and fourth degrees," Mat. Sb., v. 40 (82), 1956, pp. 123-136. (Russian) MR 19, 533.

3. K. K. BILLEVIC, "Letter to the Editor," Mat. Sb., v. 48 (90), 1959, p. 256. (Russian) MR 23 \#A879.

4. K. K. BILLEVIC, "A theorem on unit elements of algebraic fields of order $n$," Mat. Sb., v. 64 (106), 1962, pp. 145-152. (Russian) MR 29 \#1201. 
5. Z. I. BOREVIC \& I. R. SAFAREVIC, Number Theory, "Nauka", Moscow, 1964; English transl., Pure and Appl. Math., vol. 20, Academic Press, New York, 1966. MR 30 \#1080; 33 \#4001.

6. B. N. DELONE \& D. K. FADDEEV, The Theory of Irrationalities of the Third Degree, Trudy Mat. Inst. Steklov., v. 11, 1940; English transl., Transl. Math. Monographs, vol. 10, Amer. Math. Soc., Providence, R. I., 1964. MR 2, 349; 28 \#3955.

7. O. HEMER, On the Diophantine Equation $y^{2}-k=x^{3}$, Doctoral Dissertation, Uppsala, 1952.

8. O. HEMER, "Notes on the Diophantine equation $y^{2}-k=x^{3}$," Ark. Mat., v. 3, 1954, pp. 67-77. MR 15, 776.

9. H. LONDON \& R. FINKELSTEIN, On Mordell's Equation $y^{2}-k=x^{3}$, Bowling Green State Univ., Bowling Green, Ohio, 1973. MR 49 \#4928.

10. H. B. MANN, Introduction to Algebraic Number Theory, Ohio State Univ. Press, Columbus, Ohio, 1956. MR 17, 240.

11. G. F. VORONOİ, On a Generalization of the Algorithm of Continued Fractions, Doctoral Dissertation, Warsaw, 1896. (Russian)

12. H. C. WILlIAMS \& C. R. ZARNKE, A Table of Fundamental Units for Cubic Fields, Scientific Report No. 63, University of Manitoba, 1973. 